\title{
Chronic lymphocytic leukemia cells induce changes in gene expression of CD4 and CD8 T cells
}

\author{
Güllü Görgün,, ${ }^{1}$ Tobias A.W. Holderried,, ${ }^{1}$ David Zahrieh,, ${ }^{1}$ Donna Neuberg, ${ }^{1}$ and John G. Gribben ${ }^{2}$ \\ ${ }^{1}$ Department of Medical Oncology and Department of Biostatistics and Computational Biology, Dana-Farber Cancer Institute, \\ Harvard Medical School, Boston, Massachusetts, USA. ${ }^{2}$ Cancer Research United Kingdom, Department of Medical Oncology, \\ Barts and The London School of Medicine, Queen Mary University of London, London, United Kingdom.
}

\begin{abstract}
To examine the impact of tumors on the immune system, we compared global gene expression profiles of peripheral blood $T$ cells from previously untreated patients with B cell chronic lymphocytic leukemia (CLL) with those from age-matched healthy donors. Although the cells analyzed were not part of the malignant clone, analysis revealed differentially expressed genes, mainly involved in cell differentiation in CD4 cells and defects in cytoskeleton formation, vesicle trafficking, and cytotoxicity in CD8 cells of the CLL patients. In coculture experiments using CLL cells and T cells from healthy allogeneic donors, similar defects developed in both CD4 and CD8 cells. These changes were induced only with direct contact and were not cytokine mediated. Identification of the specific pathways perturbed in the $T$ cells of cancer-bearing patients will allow us to assess steps to repair these defects, which will likely be required to enhance antitumor immunity.
\end{abstract}

\section{Introduction}

Development of cancer is associated with immune suppression in the host, contributing to the failure to mount an effective immune response against the cancer cells (1). The mechanisms whereby specific $T$ cell defects occur are not well understood but include production of immune-suppressive factors by cancer cells, direct tumor cell-T cell interactions, and induction of regulatory $\mathrm{T}$ cell subsets. Identification of the specific $T$ cell defects that occur in cancer-bearing patients usually requires isolation of tumor-infiltrating lymphocytes, which limits the number of $\mathrm{T}$ cells that can be obtained for study. Tumor cells circulate in leukemia, so there is widespread interaction of cancer cells with $\mathrm{T}$ cells that can readily be sampled from peripheral blood. Specifically in B cell chronic lymphocytic leukemia (CLL), a number of well-characterized T cell defects have been described, and it is most likely that immunosuppression induced by the malignant B cells plays an important role in the induction of subsequent immune deficiency in this disease. CLL cells express high levels of immunomodulatory factors including TGF- $\beta$ and IL-10 that suppress response to antigens, T cell activation, expansion, and effector function (2-5). FasL has been detected on a number of tumors, including CLL, and FasL-positive tumor cells can induce apoptosis in vitro $(6,7)$. T cells from patients with CLL have low levels of expression of CD80, CD86, and CD154 and are Th2-preponderant (8-11). We have observed functional $\mathrm{T}$ cell defects and increased expression of Th2-type chemokine receptors on $\mathrm{T}$ cells from patients with CLL compared with $\mathrm{T}$ cells of healthy donors (12). To examine the mechanisms of $\mathrm{T}$ cell defects in tumor-bearing patients, we analyzed the global gene expression profiles of highly purified CD4 and CD8 cells from peripheral blood from individuals with CLL compared with

Nonstandard abbreviations used: CLL, chronic lymphocytic leukemia; cRNA, complementary RNA; siRNA, small interfering RNA.

Conflict of interest: The authors have declared that no conflict of interest exists.

Citation for this article: J. Clin. Invest. 115:1797-1805 (2005).

doi:10.1172/JCI24176. age-matched healthy donors. Similar defects requiring cell-cell contact were induced by coculture of healthy T cells with CLL cells. Therefore, contact with leukemic cells induces specific changes in both CD4 and CD8 T cells, resulting in functional impairment.

\section{Results}

Gene expression profiling of CD4 and CD8 T cells from CLL patients and healthy donors. CD4 and CD8 cells were isolated from healthy donors and from previously untreated patients with B cell CLL, who were selected to represent the heterogeneity of this disease (Table 1). Global gene expression profiles were obtained and the microarray data analyzed using both unsupervised and supervised learning. Even though the cells being analyzed were not part of the malignant clone, in an unsupervised analysis, delineation of patients from healthy donors was possible in all cases using hierarchical clustering of CD8 $\mathrm{T}$ cells, and in the majority of cases using hierarchical clustering of CD4 T cells (see Supplemental Figure 1; supplemental material available online with this article; doi:10.1172/JCI24176DS1).

In supervised analyses, there were no significant differences between gene expression profiles of CD4 or CD8 T cells from patients with CLL and gene expression profiles of CD4 or CD8 T cells from healthy donors, based on cell purity (less than $85 \%$ versus $85 \%$ or more), time from diagnosis ( $1-5$ years versus $6-10$ years), absolute white blood cell count (less than $20 \mathrm{~mm}^{3}$ versus $20 \mathrm{~mm}^{3}$ or more), stage of disease (0-I versus II-III), Ig heavy chain mutational status (mutated versus unmutated), or cytogenetic abnormalities (deletion $13 q$ versus others). The majority of the contaminating cells in the $\mathrm{T}$ cell population were CD19 B cells.

Molecular defects in CD4 cells in tumor-bearing patients. By supervised analysis of CD4 cells, we identified 22 genes that had significantly increased expression and 68 genes that had significantly decreased expression $(P<0.05)$ in CD 4 cells of CLL patients $(n=22)$ compared with healthy donors $(n=12)$ (Figure 1A). The differentially expressed genes were classified by their involvement in specific cellular pathways, and the full listing of these differentially expressed 


\section{Table 1}

Patients' clinical disease characteristics

\begin{tabular}{|c|c|c|c|c|c|c|c|c|c|c|c|c|c|c|}
\hline \multirow[b]{2}{*}{ Sex } & \multicolumn{6}{|c|}{ Rai stage of disease } & \multicolumn{2}{|c|}{$\begin{array}{c}\lg V_{\mathrm{H}} \\
\text { mutation status }\end{array}$} & \multicolumn{6}{|c|}{ Cytogenetics by FISH } \\
\hline & Age & Time from & Stage & Stage & Stage & Stage & UM & M & Normal & Deletion & Deletion & Deletion & $\begin{array}{c}\text { Trisomy } \\
12\end{array}$ & $\mathrm{~T}(5 ; 14)$ \\
\hline F $\quad \mathbf{M}$ & & diagnosis & 0 & I & II & III & & & & $13 q$ & $11 q$ & $17 p$ & 12 & \\
\hline 1415 & $40-80 \mathrm{yr}$ & $1-10 \mathrm{yr}$ & 8 & 14 & 5 & 2 & 7 & 22 & 12 & 11 & 1 & 1 & 3 & 1 \\
\hline
\end{tabular}

CD4 and CD8 cells were obtained from peripheral blood of patients with CLL. The patients were untreated and were chosen to represent the heterogeneity of this disease. Healthy donors were age matched. UM, unmutated; M, mutated.

genes is shown in Supplemental Table 1. The majority of the genes were involved in cell differentiation and proliferation, survival, cytoskeleton formation, and vesicle trafficking. For genes selected as representative of the defective pathways, changes in RNA expression were confirmed by real-time PCR and changes in protein expression by Western blot (Figure 2, A and B).

In the CD4 cells of CLL patients, there was decreased expression in a number of genes in the Ras-dependent JNK and p38 MAPK pathways. The JNK-p38 MAPK pathway plays major roles in CD4 $\mathrm{T}$ cell differentiation into Th1 or Th2 subsets (13-15). There was decreased gene expression in a number of components of this pathway, including the activator MINK (MAP4K6) $(16)$; GDI1 $(17,18)$, which serves as a negative regulator of small GTP-binding proteins in the Ras-dependent MAPK pathway in induction of NF-KB or actin cytoskeleton remodeling via the Arp $2 / 3$ complex; and NFRKB, which binds to several of the $\mathrm{\kappa B}$ regulatory elements $(17,19,20)$ (Figure 1B). There was also decreased expression of PIK3CB, a regulator of cell growth in response to various mitogenic stimuli through TCR/CD28, IL-1 receptor, G-protein coupled receptor, and members of the TNF receptor family $(20,21)$.

Differential expression of genes involved in cytoskeleton formation and vesicle trafficking in CD4 cells from CLL patients included decreases in $A A K 1$, which plays a regulatory role in cell migra- tion and clathrin-mediated endocytosis (22), and AP3M2, which facilitates budding of vesicles from the Golgi membrane and trafficking to lysosomes (23). There was increased expression, in CD4 cells from CLL patients, of SPTBN1; of ARPC1, which encodes an actin cytoskeleton-associated protein that plays a role in cell migration/motility or cytokine production/secretory functions by controlling actin polymerization; and of ADIR (Figure 1B).

Functionally, these changes would be expected to result in decreased Th1 differentiation, and we and others have previously demonstrated skewing of $\mathrm{T}$ cell responses to Th2 rather than Th1 differentiation in patients with CLL $(12,24)$.

Molecular defects in CD8 T cells in patients with CLL. By supervised analysis, a larger number of genes $(n=273)$ had deregulated expression in CD8 cells, including 105 genes that were downregulated and 168 genes upregulated in CD8 T cells from patients with CLL $(n=20)$ compared with healthy donors $(n=12)(P<0.05)$ (Figure 3A). The differentially expressed genes were classified by their involvement in specific cellular pathways, and a number of representative genes of those pathways are listed in Supplemental Table 2. On analysis of these genes, the majority were involved in cytoskeleton formation, intracellular transportation, vesicle trafficking, or cellular secretion as well as cytotoxicity pathways in CD8 T cells (Figure 3B).
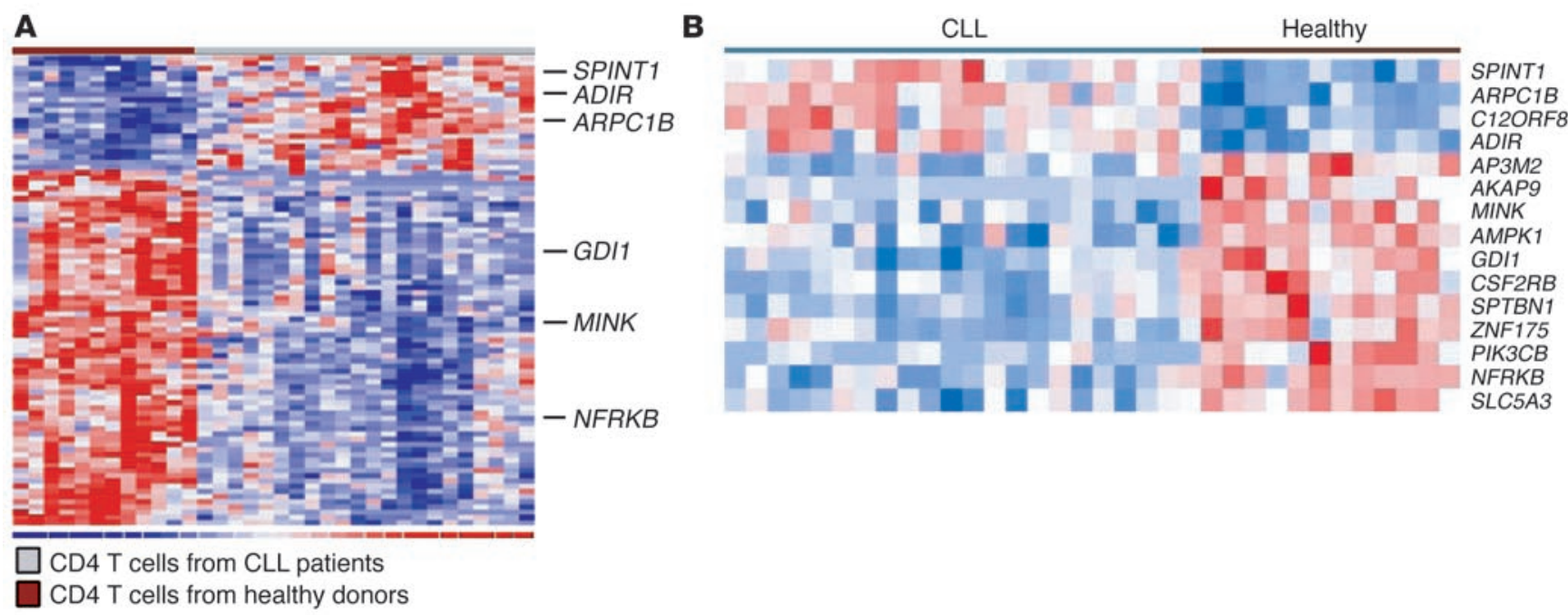

Figure 1

Differentially expressed genes in CD4 cells from patients with CLL compared with healthy donors. Dendrogram of differentially expressed genes by supervised analysis $(P<0.05)$. (A) CD4 cells from patients with CLL compared with healthy donors. Twenty-two genes were significantly increased (red) and 68 genes significantly decreased (blue) in CD4 cells from CLL patients. (B) Genes involved in Ras-dependent JNK and p38 MAPK pathways in CD4 cells. The dendrogram represents selected genes from $\mathbf{A}$. 
A

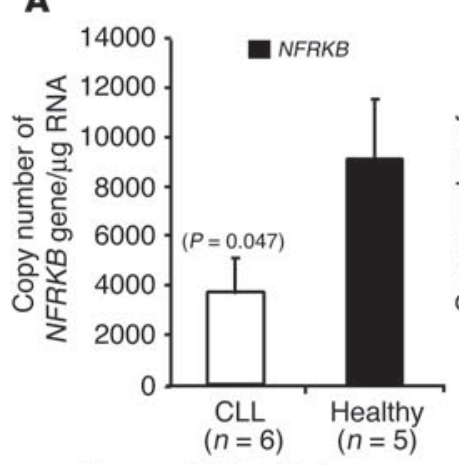

C

CD8 cells from healthy donors

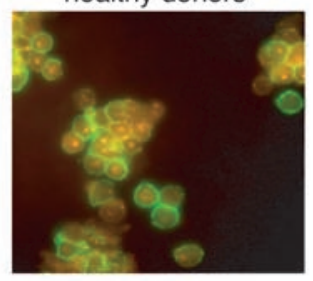

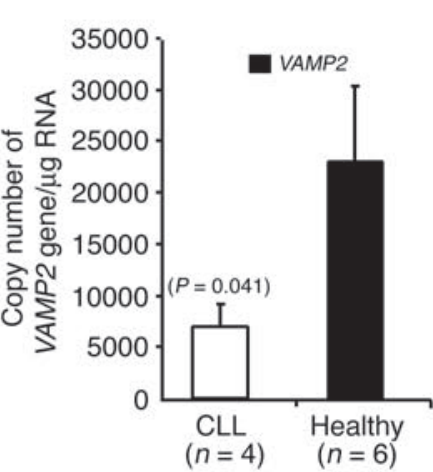

CD8 cells from CLL patients

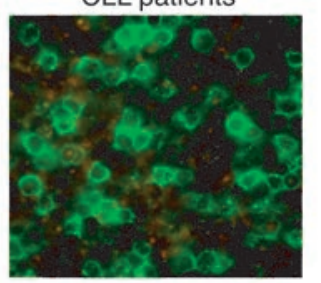

B
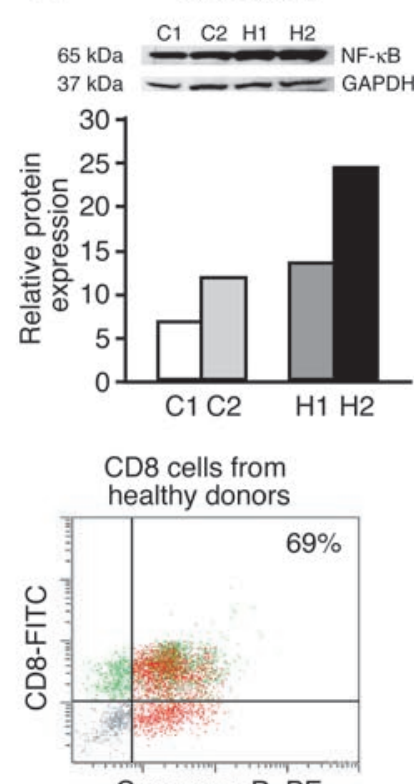

Granzyme B-PE
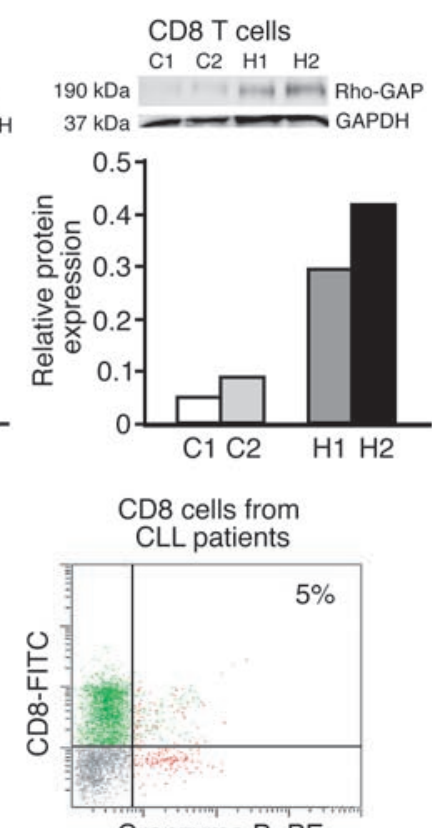

Granzyme B-PE

Figure 2

Validation of gene expression observed by microarray. (A) Concordant with data seen on microarray, by quantitative PCR there was decreased expression of NFRKB in CD4 cells and VAMP2 in CD8 cells from CLL patients compared with healthy donors. The figure represents data from CD4 cells from 6 CLL patients and 5 healthy donors and CD8 cells from 4 CLL patients and 6 healthy donors. Statistical significance was assessed in a 2-tailed Student's $t$ test. (B) Decreased expression of NF-KBp65 in CD4 cells and Rho-GAP p190 proteins in CD8 cells from CLL patients compared with healthy donors. The left 2 lanes represent protein expression in CD4 or CD8 cells from 2 CLL patients (C1 and C2), and the right 2 lanes represent 2 healthy donors $(\mathrm{H} 1$ and $\mathrm{H} 2$ ). The expression of proteins was normalized by GAPDH expression level and is shown as protein bands and densitometric intensity of each band. The figure is representative of 3 additional experiments performed on 6 different donors, all showing a similar pattern $(P<0.05)$. (C) Intracytoplasmic expression of the GP1 gene product granzyme B, detected in CD8 cells from CLL patients and healthy donors by flow cytometry and fluorescent microscopy. To obtain at least $99 \%$ CD8 cell population, cells were purified using magnetically labeled negative cell-depletion antibodies. High expression of granzyme B in CD8 cells from healthy donors (CD8-FITC+ granzyme $\mathrm{B}-\mathrm{PE}^{+}$, orange-brown) was observed compared with that in CD8 cells from CLL patients (CD8-FITC+ granzyme B-PE-, green). The figure is representative of experiments performed with 4 different patients and healthy donors $(P<0.05)$.

Impaired cytoskeleton formation, intracellular transportation, and cytotoxicity in CD8 T cells from CLL patients. We observed decreased expression of $A R A P 3$, a Rho repressor gene that induces PI3Kdependent rearrangements in the cell cytoskeleton (25); myosin $I X B$, a GTPase-activating protein for the $\mathrm{G}$ protein Rho (26); AP3M2; VAMP2; GPR57; and AKAP9. There was increased expression of CDC42, PIK4CB, RAB35, FLNA, and FMNL, which associate with both Rac and profilin and regulate reorganization of the actin cytoskeleton in association with $\operatorname{Rac}(27,28)$. Actin polymerization at the immune synapse is required for $\mathrm{T}$ cell activation and effector function, and T cell binding to APCs induces localized activation of CDC42 and WASP at the immune synapse $(29,30)$. There was increased expression of $A R P C 1 B$, required for the formation and stabilization of the immunological synapse at the interface between APCs and T lymphocytes (27-29). We also observed increased expression in SPEC1, which encodes a GTPase inhibitor protein that regulates CDC42 function, and NCK2, which encodes an src homology domain-containing (SH2 and SH3 domain-containing) adaptor protein that couples receptor tyrosine phosphorylation to downstream effector molecules in cytoskeleton formation processes (31).

There was also dysregulation of genes involved in secretory vesicle formation and cytotoxic activity. Such decreased genes included VAMP2; SCAMP1, which encodes a carrier to the cell surface in post-Golgi recycling pathways during vesicular transport; $X A B 2$, a Ras superfamily member involved in controlling a diverse set of essential cellular functions; and GPR57, a GTPbinding protein that activates JNK-, MAPK-, and p38-dependent pathways in the cytotoxic immune response (32). We observed increased expression in inhibitor genes including the Rab family members $R A B 35, R A B 22 A$, the ral guanine nucleotide dissociation stimulator RALGDS that inhibits binding of Raf to Ras, and RASGRP2, an inhibitor of guanine nucleotide exchange factor. Also increased was $A P 2 B 1$, an adaptin family member essential for the formation of adaptor complexes of clathrin-coded vesicles $(31,33)$. Adaptins interact with the cytoplasmic domains of membrane-spanning receptors in the course of their endocytic/ exocytic transport. Likely as a consequence of these changes in structural proteins, we observed a decrease in cytotoxicity in CD8 cells of CLL patients compared with healthy donors (data not shown) and a decrease in granzyme B protein in CD8 T cells of CLL patients compared with healthy donors (Figure 2C). Of note, there was no decrease in granzyme B mRNA expression in the CD8 T cells in CLL patients, and we conclude that the decreased granzyme B protein expression reflects failure to package the protein in secretory vesicles. 
A

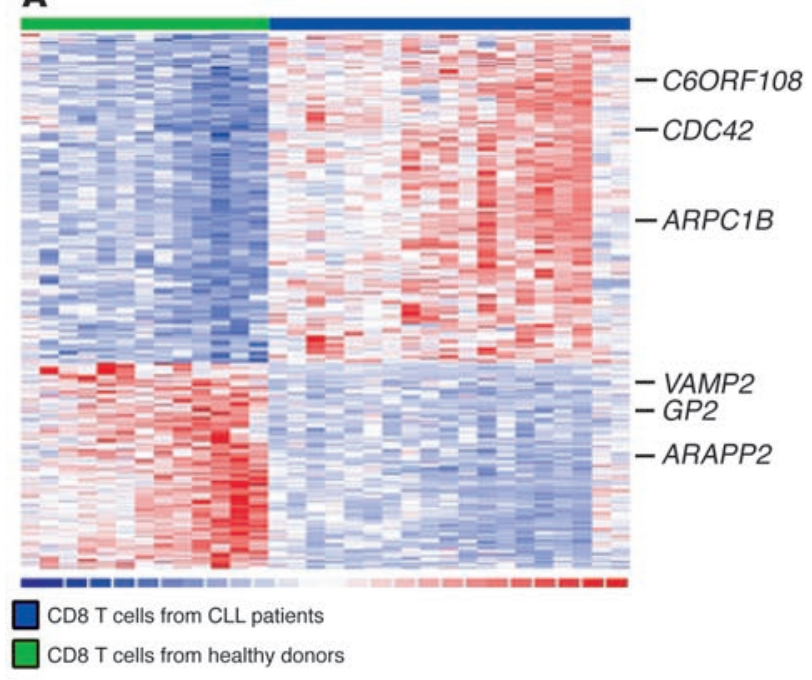

B Healthy CLL

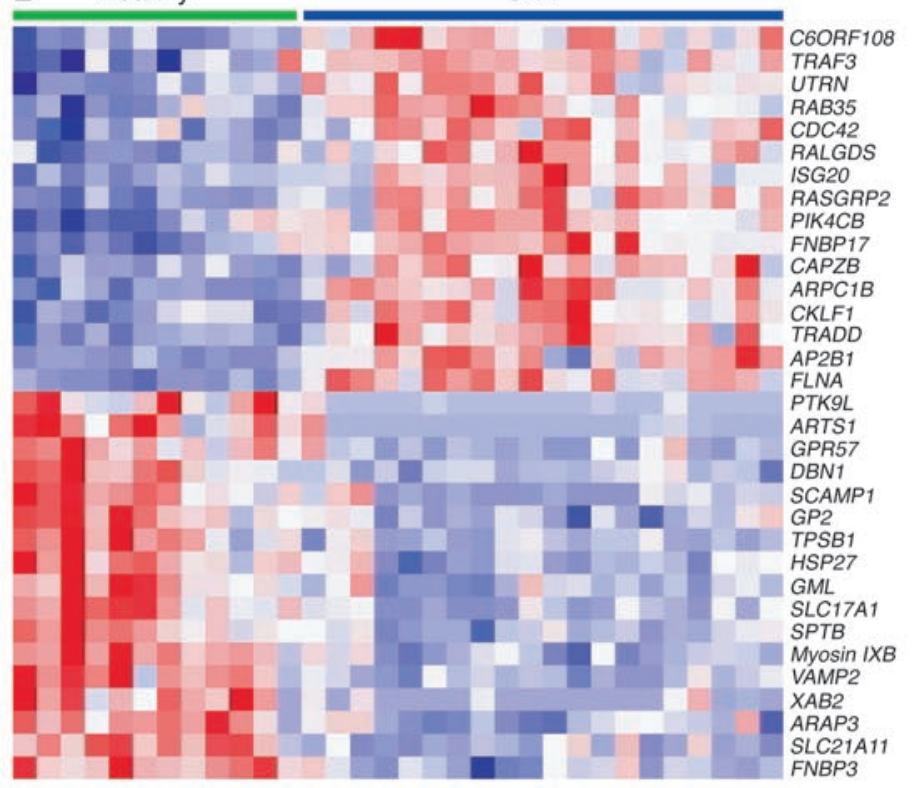

Figure 3

Differentially expressed genes in CD8 cells from patients with CLL. Dendrogram of differentially expressed genes by supervised analysis in CD8 cells from patients with CLL compared with healthy donors $(P<0.05)$. (A) One hundred sixty-eight genes were significantly increased (red) and 105 genes were significantly decreased (blue) in CD8 cells from CLL patients. (B) Dendrogram of differentially expressed genes involved in cytoskeleton formation, vesicle trafficking, and cytotoxicity pathways in CD8 cells. The dendrogram represents selected genes from $\mathbf{A}$.

These changes would be expected to result in decreased cytotoxicity and effector function. We and others have previously demonstrated that such defects occur in the CD8 T cells in patients with CLL $(12,34,35)$.

We therefore identified specific pathways with altered expression in CD4 and CD8 cells of CLL patients. From this we developed a representative protein expression panel using Western blot analysis and used this proteomic approach to assess whether CLL cells could induce similar changes in healthy allogeneic T cells and to elucidate the mechanism(s) whereby CLL cells could induce changes in these pathways, using cocultures of healthy T cells with CLL cells.

The CLL B cell-derived soluble factors induce alterations in chemokine and chemokine receptor expression but not cytoskeletal proteins in bealthy T cells. CLL cells express cytokines known to inhibit $\mathrm{T}$ cell responses, including IL-10. We therefore hypothesized that release of these inhibitory cytokines would induce the changes in gene expression observed in healthy CD4 and CD8 cells. However, following culture of healthy CD4 or CD8 cells with sera from CLL patients or coculture of CLL cells or healthy B cells with healthy CD4 or CD8 cells in transwell culture plates, we did not observe changes in expression of cytoskeleton proteins or other genes that we have shown to be decreased in CD4 or CD8 cells in CLL patients (data not shown). The only defects shown to be induced by culture of healthy $\mathrm{T}$ cells with these soluble factors were altered expression of chemokines and chemokine receptors, including decreases in CXCR1, CXCR2, and CXCR4 and increases in CXCR3, CCR4, and CCR5 in CD4 T cells from healthy donors (Supplemental Figure 2). When IL-10 mRNA expression was inhibited by transient transfection of small interfering RNA (siRNA) targeting IL-10 (Supplemental Figure 3) in B cells from both CLL patients and healthy donors or by use of neutralizing anti-IL-10 mAbs, there was no change in expression level of cytoskeletal proteins, but this blocked the changes in chemokine and chemokine receptor expression, suggesting that these alterations were indeed induced by IL-10 and not by other soluble factors (Supplemental Figure 2).

CLL B cells induce alteration in cytoskeleton formation and vesicle transportation pathways in T cells by cell-cell contact. Since soluble factors did not induce changes in healthy T cells, we cocultured CLL cells in direct contact with $\mathrm{T}$ cells from healthy donors and analyzed expression of proteins representative of the pathways found to be abnormal in the cancer-bearing patients. By 48 hours of culture of healthy donor CD4 T cells with tumor cells, we observed changes in protein expression patterns consistent with that seen in the CD4 cells of the CLL patients. Such changes included increased expression of Arp3 and decreased expression of NF-кBp65 and GDI1 (Figure 4A). Similarly, in CD8 cells, we observed changes in the expression pattern consistent with that observed by gene expression profiling, including decreased Rho-GAP and increased Arp3 and CDC42 protein (Figure 4B). Induction of these changes required cell-cell contact, and these changes were not observed after blockade of adhesion molecules using anti-CD54 and antiCD11a mAbs (Figure 4C). These changes were not induced by coculture of allogeneic $\mathrm{T}$ cells with healthy $\mathrm{B}$ cells from the donors who were HLA matched to the CLL patients.

\section{Discussion}

Microarray-based expression profiling has been used most commonly to compare and contrast heterogeneous groups of human tumors to identify expression patterns associated with prognosis and to examine altered expression in tumor cells compared with their normal cellular counterparts. Here we performed gene expression profiling on nonmalignant components in cancer-bearing 
A

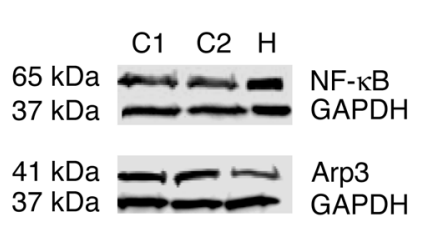

B

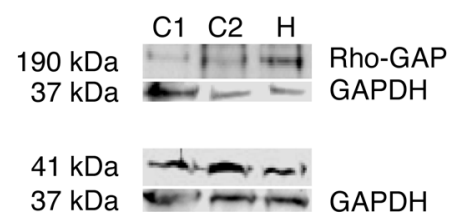

C
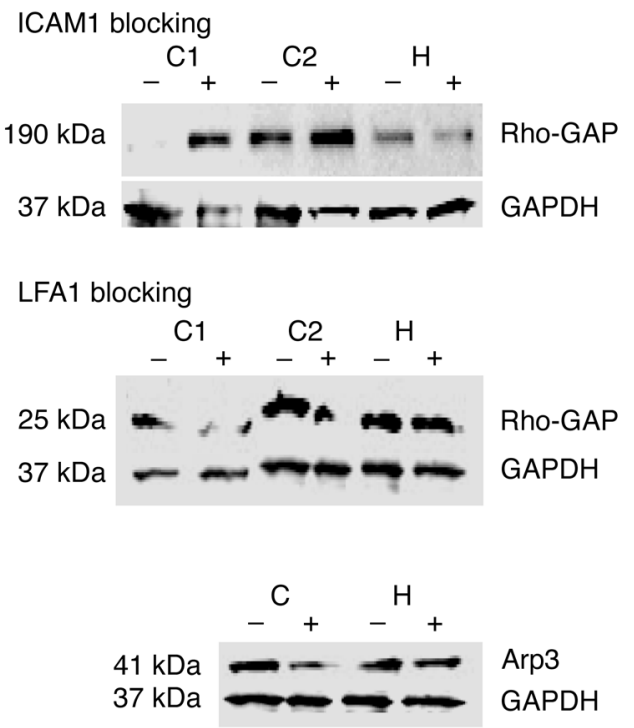

Arp3 GAPDH
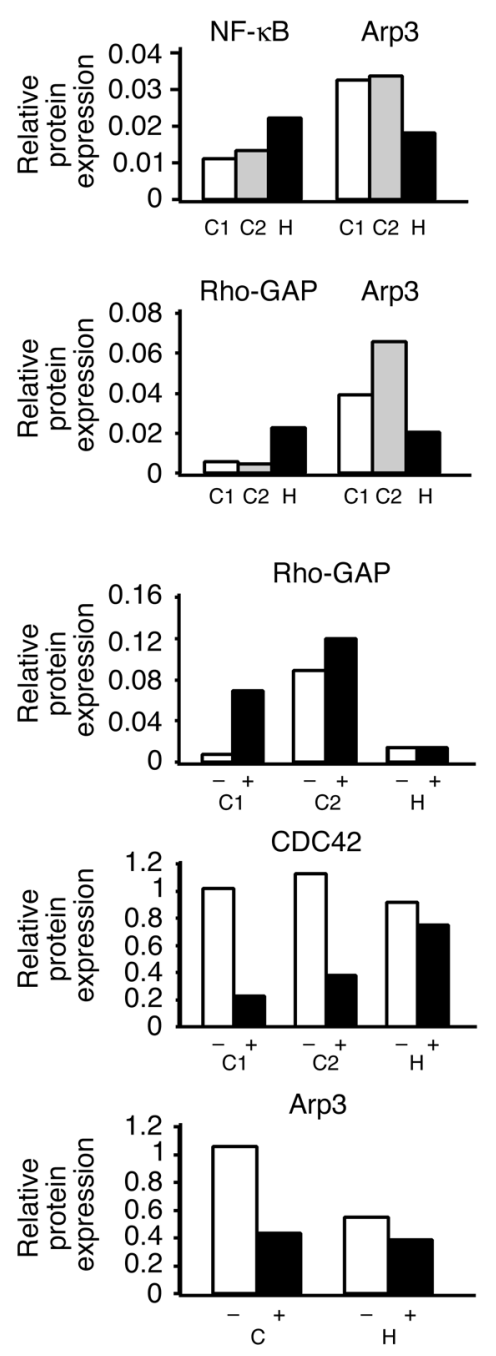

Figure 4

Impact of $\mathrm{T}$ cell-cancer cell contact on healthy $T$ cells. Highly purified $T$ cells from healthy donors were cocultured with $B$ cells from CLL patients or from their HLAmatched healthy donors at a 2:1 (T/B cell) ratio for 48 hours. (A) Decrease in $65-\mathrm{kDa}$ $\mathrm{NF}-\mathrm{kB}$ and increase in $41-\mathrm{kDa}$ Arp3 in healthy CD4 cells after contact with allogeneic CLL cells $(C 1$ and $C 2)$ and healthy $B$ cells $(H)$. (B) Decrease in 190-kDa Rho-GAP and increase in 41-kDa Arp3 in healthy CD8 cells after contact with CLL cells $(\mathrm{C} 1$ and $\mathrm{C} 2)$ and healthy allogeneic $B$ cells $(H)$. (C) Impact of CLL cell contact on cytoskeletal protein expression in allogeneic healthy T cells, confirmed by ICAM1 or LFA1 blocking. Highly purified CD4 or CD8 cells from healthy donors were cocultured with B cells from CLL patients $(\mathrm{C} 1$ and $\mathrm{C} 2)$ or healthy donors $(\mathrm{H})$ with or without blockade of the LFA1 or ICAM1 interaction. Expression of $190-\mathrm{kDa}$ Rho-GAP in healthy CD8 cells was increased after blockade of the ICAM1 during CLL cell-T cell contact, and expression of $41-\mathrm{kDa}$ Arp3 in CD4 T cells and 25-kDa CDC42 in healthy CD8 cells was decreased after blockade of the LFA1 or ICAM1. -, protein expression in nonblocked cells; + , protein expression in blocked cells. Protein expressions were normalized by GAPDH expression level and are shown as protein bands and densitometric intensity of each band. The figure is representative of 3 different experiments performed with 6 different patients with CLL and 6 different healthy donors showing a similar pattern $(P<0.05)$. patients and demonstrate profound changes in gene expression of T cells in patients with CLL compared with healthy donors. Importantly, we demonstrate that these changes can be induced at the protein level in healthy $\mathrm{T}$ cells following short-term culture with direct contact with CLL cells.

Analysis of the differentially expressed genes in the $T$ cells in CLL patients demonstrates a number of abnormalities in specific pathways. In CD4 cells, among the most marked changes observed were in the Ras-dependent JNK and p38 MAPK pathways (Figure 5). JNK and p38 MAPK pathways play a major role in regulating CD4 $\mathrm{T}$ cell differentiation into Th1 or Th2. JNK2 and 38 MAPKs mediate IFN- $\gamma$ production and Th 1 cell differentiation, and inhibition of p38 MAPK in dnp38 transgenic mice results in decreased IFN- $\gamma$ production by Th1 cells $(15,36,37)$. $A D I R$ encodes a protein involved in protein processing in the endoplasmic reticulum and contains a putative IFN-responsive ATP-binding site involved in regulating expression of genes critical for antigen presentation and immune surveillance against viruses and tumor cells (38). Our data, demonstrating decreased expression in the p38 MAPK pathway activator genes such as MINK, NFRKB, and PIK3CB, are in keeping with our hypothesis that the defects induced by the leukemic cells impair subsequent CD4 differentiation into Th1 cells.
In CD8 cells, our findings are in keeping with the hypothesis that cell contact with CLL cells induces changes in gene expression in genes regulating cytoskeleton formation and vesicle trafficking (Figure 5), thereby resulting in the decreased cytotoxicity and effector function noted in this disease. The cytoskeleton is a cellular network of structural, adaptor, and signaling molecules that regulates most cellular functions during immune responses, including migration, extravasation, antigen recognition, activation, and phagocytosis. CD8 cytotoxic T lymphocytes mediate killing of cancer cells through polarized delivery of vesicles referred to as lytic lysosomes that contain apoptosis-inducing proteins including perforin and granzymes (39-41). Positioning of the secretory cleft and secretory lysosome polarization targeting cancer cells depend on cytoskeletal connections that regulate granule transport to the plasma membrane (40). The altered expression in regulator genes, including increased $R A B 11 B$ and $R A B 22 A$ and decreased $R A B 35, V A M P 2$, SLC21A11, and SCAMP1, indicated defects in vesicle formation and intracellular trafficking in CD8 cells in CLL patients. We observed decreased expression of GP2 (41), and TPSB1, a gene encoding a tetrameric serine protease, concentrated and stored selectively in secretory granules $(40,42)$. In CD8 also we observed defects in the p38 MAPK pathway, which also regulates the production of TNF- $\alpha$, perforin, and granzyme as well as apoptosis in CD8 cells (43-45). 


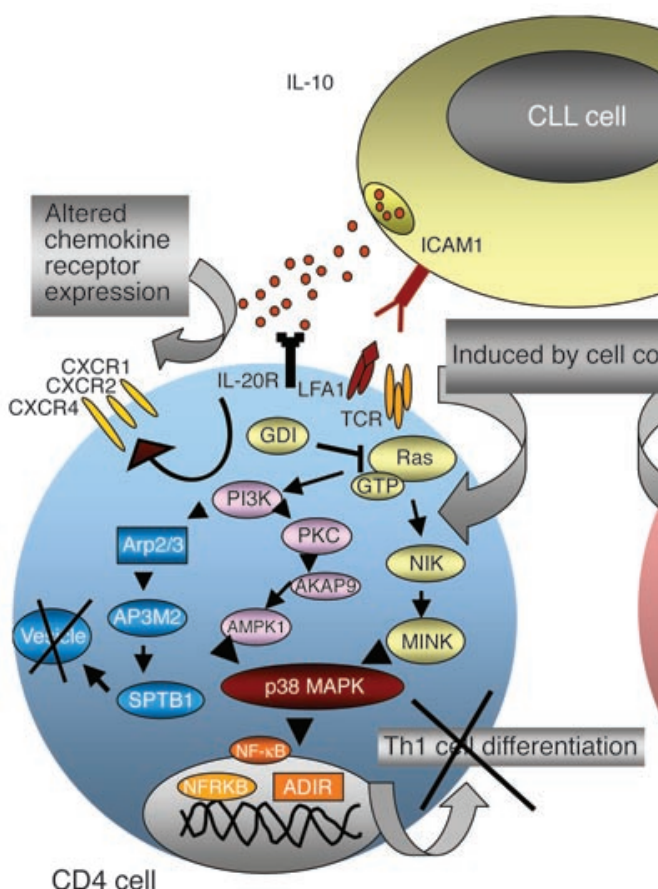

CD4 cell

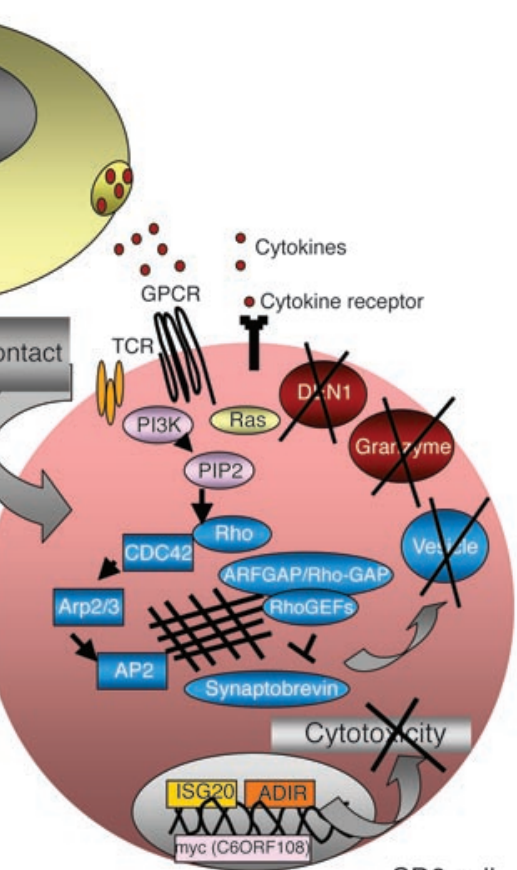

CD8 cell

\section{Figure 5}

Differentially expressed genes by their involvement in specific signaling pathways. Representative defects in T cell pathways and functions caused by CLL cell-T cell contact are shown in this diagram. Differentially expressed genes involved in cell differentiation, particularly JNK (pink) and p38 MAPK (yellow) pathways and cytoskeleton formation and vesicle transportation (blue), in CD4 T cells from CLL patients compared with healthy donors are represented by selected genes that were increased (rectangles) or decreased (ovals). Differentially expressed genes involved in cytoskeleton formation, vesicle trafficking (blue), and cytotoxicity (red) in CD8 T cells from CLL patients compared with healthy donors are represented by selected increased genes (rectangles) and decreased genes (ovals).

Our data suggest that even though CD8 T cells in CLL appear morphologically intact, the production of cytolytic molecules including granzymes and their storage in lysosomes as well as intracellular secretory vesicle transportation are significantly impaired. The decreased expression in activators and increased expression in repressor genes involved in cytoskeleton formation and intracellular vesicle transportation, more specifically the decreased expression in granzyme granules GP2 and TPSB1, likely contribute to the failure of CD8 $\mathrm{T}$ cell responses against tumor cells in CLL.

Several studies have shown that CLL cells secrete IL-10, TNF- $\alpha$, and TGF- $\beta(2-5,46)$. The inhibitory cytokine IL-10 initiates a wide variety of activities on binding to its cellular receptor complex. The mechanism of IL-10 inhibition of cytokine production was initially believed to be inhibition of the antigen-presentation capacity of macrophages and DCs (47), but IL-10 also plays important roles in blocking cytokine production, expression of costimulatory molecules, and chemokine secretion. It also modifies chemokine receptor expression, increases integrin ligand (e.g., ICAM1) expression $(48,49)$, and induces CCR5 expression on monocytes (50). Therefore IL-10 appeared an attractive candidate to induce specific changes in gene expression in T cells in CLL patients. Our results suggest that such changes are largely limited to changes in chemokine expression, but the additive effect of IL-10 production on the changes that are induced by direct contact and in vivo in a murine model is currently under investigation.
Taken together, the results presented here demonstrate that contact with cancer cells can induce changes in gene expression in healthy cells in the cancerbearing patient. These changes likely contribute to the decreased immune responses observed in these patients and ostensibly may contribute to the lack of autologous antitumor responses. We are currently studying the impact of tumor development in vivo on $\mathrm{T}$ cell function and expression profiles using the E $\mu$-TCL-1 transgenic mouse model of CLL (51). As these mice develop leuke$\mathrm{mia}$, there are changes in expression profiles of their CD4 and CD8 cells similar in nature to those observed in patients with CLL (data not shown). Moreover, the observation that CLL cells are capable of inducing similar changes in allogeneic $\mathrm{CD} 4$ and $\mathrm{CD} 8$ cells has implications for the field of allogeneic stem cell transplantation. As we have observed in the in vitro assay systems, infusion of donor $\mathrm{T}$ cells in patients with high tumor burden could induce similar changes in donor $\mathrm{T}$ cells with resulting decrease in antitumor immunity, thereby limiting the graft-versus-leukemia effect. Characterization of these defects will now allow us to examine mechanisms to repair $\mathrm{T}$ cell function to increase antitumor immunity in both the allogeneic and the autologous setting.

\section{Methods}

Cell isolation and RNA extraction. Heparinized venous blood samples from 29 CLL patients with Rai stages varying from 0 to 3 (Table 1) and age- and HLAmatched healthy donors were obtained after written informed consent. The studies using peripheral blood sample collection from all individuals were approved by the Institutional Review Board of the Dana-Farber Cancer Institute. None of the CLL patients had received chemotherapy before the blood was drawn for these studies. Mononuclear cells were separated by Ficoll-Hypaque density gradient centrifugation, and CD4 T cells from 22 patients with CLL and 12 healthy donors, CD8 T cells from 20 patients with CLL and 12 healthy donors, and normal and malignant B cells were negatively selected by depletion of the following as appropriate: CD4 or CD8 $\mathrm{T}$ cells, B cells, monocytes, granulocytes, platelets, early erythroid precursor cells, and NK cells. For negative selection, a magnetically labeled cocktail of hapten-modified anti-CD14, -CD16, -CD36, -CD56, -CD123, -TCR $\gamma \delta$, and -glycophorin A, with or without CD4, CD8, or CD19 mAbs (Miltenyi Biotec), was used. The purity of the isolated $T$ cells and $B$ cells was detected using anti-CD19, anti-CD4, and anti-CD8 antibodies. Frozen or freshly isolated CD4 or CD8 T cells were lysed in TRIzol for total-RNA isolation (Invitrogen Corp.), and 3-15 $\mu \mathrm{g}$ of total RNA was used for gene chip array.

Gene chip array. Quality control of the RNA samples was performed by spectrophotometric analysis to confirm the concentration and to detect contaminating proteins and other molecules, and a size fractionation procedure using a microfluidics instrument (Agilent Technologies) was used to determine whether the RNA was intact. 
RNA conversion of cDNA and subsequent hybridization to gene arrays were performed in the Core Facility at Dana-Farber Cancer Institute, all steps according to the manufacturer's protocols (Affymetrix Inc.). Briefly, RNA was converted into cDNA using a T7 promoter-tailed oligo-dT primer in the synthesis of the first cDNA strand, and second-strand cDNA synthesis was then carried out. The double-stranded cDNA was used as the template in an in vitro transcription (IVT) reaction catalyzed by $\mathrm{T} 7$ polymerase and containing biotinylated CTP and UTP in addition to the 4 unmodified ribonucleoside triphosphates. The biotinylated complementary RNA (cRNA) was purified from the IVT reaction mixture using the RNeasy system (QIAGEN). Purified cRNA was fragmented in order to facilitate the subsequent hybridization step. The CRNA was purified from the fragmentation reaction using phenol/chloroform extraction and ethanol precipitation. The fragmented cRNA was added to a hybridization solution containing several biotinylated control oligonucleotides and hybridized to an Affymetrix Inc. U133A microarray chip overnight at $45^{\circ} \mathrm{C}$. The chips were then washed to remove cRNA that had not hybridized to its complementary oligonucleotide probe. The bound cRNA was fluorescently labeled using PE-conjugated streptavidin (SAPE); additional fluors were then added using biotinylated anti-streptavidin antibody and additional SAPE. Each cRNA bound at its complementary oligonucleotide was excited using a confocal laser scanner, and the positions and intensities of the fluorescent emissions were captured. These measures provided the basis of subsequent biostatistical analysis.

Biostatistical analysis. Gene expression profiling was performed on peripheral blood CD4 and CD8 T cells from 29 previously untreated CLL patients and 25 healthy donors. To identify the genes whose expression patterns best distinguished CLL CD 4 and CD8 T cells from healthy CD4 and CD8 T cells, the permutation distribution of the maximum $t$ statistic was analyzed using the permax test (52). The customized program Permax 2.1, written by Robert Gray, calculates Permax values and is available free online (http://biowww.dfci.harvard.edu/ gray/permax. html). Within the CLL CD4 and CD8 T cells we compared gene expression profiles using the permax test according to cell purity (less than $85 \%$ versus $85 \%$ or more), time from diagnosis (1-5 years versus $6-10$ years), absolute white blood cell count (less than $20 \mathrm{~mm}^{3}$ versus $20 \mathrm{~mm}^{3}$ or more), stage of disease (0-I versus II-III), Ig heavy chain mutational status (mutated versus unmutated), and cytogenetic abnormalities (deletion $13 \mathrm{q}$ versus others). In these supervised analyses, the permax $P$ value was calculated by comparison of the observed $t$ statistics to the permutation distribution of the largest $t$ statistic obtained over the 22,283 genes. For each gene, the $P$ value is the proportion of permutations with the maximum $t$ statistics over all genes greater than or equal to the observed value for a particular gene. A test declaring as significant any genes with $P$ less than 0.05 guarantees that the chance of any false positives being selected is less than $5 \%$. In our analysis, permax $P$ values less than 0.05 were deemed statistically significant.

DNA-Chip Analyzer (dChip) (53) was used to normalize the Affymetrix gene array data and to obtain perfect-match-only model-based expression intensities. An array with a median overall intensity was chosen as the baseline array against which other arrays were normalized at probe intensity level. dChip was used to perform an unsupervised analysis that consisted of gene filtering, that is, exclusion of genes that lacked sufficient variability across groups, and hierarchical clustering of genes and samples. The filtering criteria required that a gene's coefficient of variation across all samples (after pooling of replicate arrays) be between 0.4 and 10. In addition, the filtering criteria required that a gene be called "present" in more than $20 \%$ of the arrays. Spearman's rank correlation was used to assess the correlation between the quantitative PCR analysis and Affymetrix data of genes from selected pathways.
Quantitative RT-PCR. Selected genes were analyzed for expression by realtime, quantitative PCR using SYBR Green labeling (Applied Biosystems). To confirm the gene expression level detected by microarray, the expression of 8 selected genes was measured in CD4 cells from 6 patients with CLL and 5 healthy donors and CD8 cells from 4 patients with CLL and 6 healthy donors. To confirm the inhibition of siRNA-targeted mRNA, the expression of IL-10 and vimentin relative to $18 \mathrm{~S}$ was measured in $\mathrm{B}$ cells from 6 patients with CLL. cDNA was synthesized by $400 \mathrm{U}$ Moloney murine leukemia virus reverse transcriptase from $2 \mu \mathrm{g}$ of total RNA in the presence of $1 \times$ PCR buffer, $1 \mu \mathrm{M}$ oligo-(dT) 18 primer, $2 \mu \mathrm{M} \mathrm{dNTP}$, and $40 \mathrm{U}$ RNase inhibitor according to the manufacturer's instructions (Advantage RT-for-PCR kit; BD Biosciences - Clontech).

Primers targeting the region of the Affymetrix probe were designed using Primer Express 1.0 software (Applied Biosystems) for NFRKB (forward 5'-GGGATCTTGATTGTGGCCG-3' and reverse 5'-AACAGGCTTGCCAAGAAGGA-3'), for VAMP2 (forward 5'-GAGACATGACCAGGAAGCCAA-3' and reverse 5'-CCCTAATCCTCTGAGCCTCTGA$3^{\prime}$ ), for $I L-10$ (forward $5^{\prime}$-TTCCAGTGTCTCGGAGGGA-3' and reverse 5'-GGCCACAGCTTTCAAGAATG-3'), for vimentin (forward 5'-GCATGTCCAAATCGATGTGG-3' and reverse 5'-CGTACGTCACGCAGGGC$3^{\prime}$ ), and for 18S rRNA (forward 5'-AGTCCCTGCCCTTTGTACACA-3' and reverse $5^{\prime}$-CGATCCGAGGGCCTCACTA-3'). After the optimization, $20 \mathrm{ng}$ cDNA was used as target DNA in the presence of $12.5 \mu \mathrm{l} \mathrm{SYBR}$ Green PCR Master Mix (Applied Biosystems) under optimal conditions. The amplification profile for all genes was 40 cycles of $50^{\circ} \mathrm{C}$ for $2 \mathrm{~min}$ utes, $95^{\circ} \mathrm{C}$ for 10 minutes, $95^{\circ} \mathrm{C}$ for 15 seconds, and $56^{\circ} \mathrm{C}$ for $30 \mathrm{sec}$ onds. The specificity of PCR products was analyzed by performance of a melting curve $\left(1 \mathrm{cycle}\right.$ of $60^{\circ} \mathrm{C}$ for 15 seconds, $95^{\circ} \mathrm{C}$ for 20 seconds, and $60^{\circ} \mathrm{C}$ for 15 seconds) followed by analysis on Dissociation Curve Analysis 1.0 software (Applied Biosystems). For absolute-quantitation purposes, PCR products were cloned into TOPO vector using a TOPO TA Cloning kit (Invitrogen Corp.), and standard curves were performed to determine copy numbers of genes.

Detection of intracytoplasmic granzyme B. Peripheral blood CD8 cells were purified from CLL patients and healthy donors using magnetically labeled negative cell-depletion antibodies against CD4, CD14, CD16, CD19, CD36, CD56, CD123, TCR $\gamma \delta$, and glycophorin A. To inhibit granzyme B secretion, CD8 T cells, at least $99 \%$ pure, were incubated in culture medium (10\% human $\mathrm{AB}$ serum, $1 \%$ penicillin-streptomycin, $1 \%$ L-glutamine) containing $5 \mu \mathrm{g} / \mathrm{ml}$ of brefeldin-A solution (eBioscience) for 2 hours at $37^{\circ} \mathrm{C}$. Cells were stained with FITC-conjugated anti-CD8 antibody (BD Biosciences). Cells were fixed in $4 \%$ paraformaldehyde-PBS and stained with PE-conjugated anti-granzyme B antibody (Sanquin Reagents) in permeabilization buffer (0.5\% saponin-PBS). Intracytoplasmic expression of granzyme B in CD8 cells was detected by flow cytometry (Beckman Coulter Inc.) and fluorescein microscopy (AX70; Olympus Corp.).

Western immunoblot. Translational gene expression level was measured by Western immunoblot. Total cell protein was extracted from at least 99\% pure CD4 T or CD8 T cells isolated by depletion of non-CD4 or nonCD8 $\mathrm{T}$ cells. $\mathrm{T}$ cells were lysed with radioimmuno-precipitation assay buffer (RIPA) lysis buffer containing protease inhibitors and boiled in SDS-loading buffer for 5 minutes before analysis by SDS-PAGE. Proteins were separated by SDS-PAGE and transferred onto PVDF membrane. Protein blots were performed with the following primary antibodies: rabbit anti-human CDC42, -human Arp2, -human Arp3, or -human PI3K (Santa Cruz Biotechnology Inc.), rabbit anti-human RhoGDI or -human NF-кBp65 (Upstate), or mouse anti-human Rho-GAP (Upstate). Proteins then were labeled with secondary HRP-conjugated goat anti-rabbit Ig antibody and detected by Western blot chemiluminescence reagents (PerkinElmer). The expression levels of proteins were 
normalized with GAPDH (Santa Cruz Biotechnology Inc.) expression and were measured using a Kodak Digital Science Image Station 440CF luminometer (Eastman Kodak Co.).

B cell-T cell interaction. B cells and CD4 and CD8 T cells were isolated by negative magnetic cell depletion from previously untreated patients with CLL or healthy donors. The cell purity was at least $99 \%$ CD4, CD8, or CD19 by flow cytometry. To assess the tumor cell-derived soluble effect on T cells in CLL, $2.5 \times 10^{6} \mathrm{CD} 4$ or CD8 T cells per milliliter from healthy donors were incubated for 48 hours in the presence of $10 \%$ sera obtained from patients with CLL or healthy donors. Healthy CD4 or CD8 T cells $\left(3 \times 10^{6}\right.$ per milliliter $)$ were incubated with $1.5 \times 10^{6} \mathrm{~B}$ cells per milliliter from patients with CLL or healthy donors in culture medium on 24-well transwell plates for 48 hours as well.

To analyze the impact on T cells of tumor cell contact, $2.5 \times 10^{6} \mathrm{~B}$ cells per milliliter from CLL patients or healthy donors were incubated with $5 \times 10^{6}$ healthy $\mathrm{CD} 4$ or $\mathrm{CD} 8 \mathrm{~T}$ cells per milliliter at a 1:2 ratio (B/T cells) in culture medium for 48 hours. After incubation, cells were harvested and T cells isolated by magnetic cell separation. The purity of $\mathrm{T}$ cells was detected using fluorescence-conjugated anti-CD4 or -CD8 antibodies. Total cell protein was extracted from $T$ cells by lysing of cells in RIPA lysis buffer. To analyze further the impact on T cells of CLL cell-derived IL-10, IL-10 expression in CLL cells was inhibited by IL-10 siRNA transfection. Autologous CD4 or CD8 cells from CLL patients were cocultured with IL-10 siRNA-treated or nontreated CLL cells at a 2:1 (T/B cell) ratio for 24-48 hours in culture medium.

IL-10, CD54, and CD11a/CD18 blocking. Ten percent sera from patients with CLL or healthy donors were incubated in the presence of $2 \mu \mathrm{g} / \mathrm{ml}$ of anti-human IL-10 (BD Biosciences - Pharmingen) neutralizing antibody in culture medium for 30 minutes. T cells $\left(3 \times 10^{6}\right.$ per milliliter $)$ from healthy donors were added and incubated for 48 hours at $37^{\circ} \mathrm{C}$. B cells and $\mathrm{T}$ cells were cocultured in the presence or absence of blocking antibodies for 48 hours. Anti-IL-10 neutralizing antibody $(2 \mu \mathrm{g} / \mathrm{ml})$, anti-CD54 $(100 \mu \mathrm{g} / \mathrm{ml})$, and/or anti-CD11a/CD18 $(50 \mu \mathrm{g} / \mathrm{ml})$ blocking antibodies (R\&D Systems) were added to $\mathrm{B}$ cells $\left(1.5 \times 10^{6}\right.$ per milliliter $)$ or $\mathrm{T}$ cells $\left(3 \times 10^{6}\right.$ per milliliter $)$ for 1 hour in either 24 -well plates or $0.45-\mu \mathrm{m}$ pore transwell plates. Following blocking, $\mathrm{CD} 4$ or $\mathrm{CD} 8 \mathrm{~T}$ cells $\left(3 \times 10^{6}\right.$ per milliliter $)$ from healthy donors were cocultured with CLL B cells or healthy B cells for 48 hours in the absence or presence of blocking antibodies. T cells were harvested, cytoskeletal protein expressions were measured by Western blot, and cell surface chemokine and chemokine receptor expressions were analyzed by flow cytometry.

Flow cytometry. After cell-cell interaction, T cells were stained with anti-human CD4-FITC or anti-human CD8-FITC mAbs; CXCR1-PE, CXCR3-PE, CXCR4-Cy chrome, CCR4-PE, and CD4- or CD8-PE mAbs; and CXCR2-FITC mAbs (BD Biosciences - Pharmingen). The purity of iso- lated cell populations was detected using fluorescence-labeled anti-human CD19, -human CD20, -human CD4, and -human CD8 mAbs (BD Biosciences - Pharmingen, and Beckman Coulter Inc.).

siRNA transfection and gene silencing. Highly purified (at least 99\% pure) CLL cells were plated onto 24 -well plates at $10 \times 10^{6}$ per milliliter 24 hours before transfection in culture medium. On the day of transfection, cells were transfected with $1.5 \mu \mathrm{g}$ nonspecific control siRNA targeting AATTCTCCGAACGTGTCACGT, sense r(UUCUCCGAACGUGUCACGU)dTdT, and antisense r(ACGUGACACGUUCGGAGAA)dTdT; positive control siRNA targeting vimentin AAGAATGGTACAAATCCAAGT, sense r(GAAUGGUA CAAAUCCAAGU)dTdT, and antisense r(ACUUGGAUUUGUACCAUUC)d TdT; and siRNA targeting human IL-10 CAGAGTGAAGACTTTCTTTCA, sense 5'-GAGUGAAGACUUUCUUUCAd(TT)-3', and antisense $3^{\prime}$ d(GT)CUCACUUCUGAAAGAAAGU-5' (QIAGEN), by electroporation according to the manufacturer's protocol (Amaxa GmbH). Cells were resuspended in $1 \mathrm{ml}$ of culture medium and incubated for 48 hours. Transfection efficiency was detected by transfection of FITC-conjugated control siRNA and visualized by fluorescein microscopy. The silencing efficiency was analyzed at mRNA expression level by quantitative PCR using vimentin-specific primers as described above. The relative expression of vimentin or IL-10 mRNA in CLL cells was determined by normalizing to $18 \mathrm{~S}$ mRNA expression level. Furthermore, the silencing efficiency of IL-10 siRNA was analyzed at protein expression level by Western blot. Relative IL-10 protein expression in CLL cells treated with nonspecific control siRNA or IL-10 siRNA was detected with anti-human IL-10 polyclonal antibody (Santa Cruz Biotechnology Inc.) and normalized to GAPDH protein level.

\section{Acknowledgments}

We thank Peter Varney for editorial assistance, and Charles Lee, Edward Alvin Fox, and the staff of the Dana-Farber Cancer Institute Microarray Core Facility. This work was supported by grant PO1 CA81538 from the National Cancer Institute to the CLL Research Consortium (J.G. Gribben and D. Neuberg) and the Eskandarian Family CLL Research Fund.

Received for publication December 13, 2004, and accepted in revised form May 10, 2005.

Address correspondence to: John G. Gribben, Cancer Research United Kingdom Medical Oncology Unit, St. Bartholomew's Hospital, Barts and The London School of Medicine, Charterhouse Square, London EC1M 6BQ, United Kingdom. Phone: 44-207-882-6126; Fax: 44-207-882-6004; E-mail: john.gribben@cancer.org.uk.
1. Houghton, A.N., and Guevara-Patino, J.A. 2004 Immune recognition of self in immunity against cancer. J. Clin. Invest. 114:468-471. doi:10.1172/ JCI200422685.

2. Benjamin, D., Knobloch, T.J., and Dayton, M.A. 1992. Human B-cell interleukin-10: B-cell lines derived from patients with acquired immunodeficiency syndrome and Burkitt's lymphoma constitutively secrete large quantities of interleukin-10. Blood. 80:1289-1298.

3. Benjamin, D., Park, C.D., and Sharma, V. 1994. Human B cell interleukin 10. Leuk. Lymphoma. 12:205-210.

4. Lotz, M., Ranheim, E., and Kipps, T.J. 1994. Transforming growth factor beta as endogenous growth inhibitor of chronic lymphocytic leukemia B cells. J. Exp. Med. 179:999-1004.

5. Fayad, L., et al. 2001. Interleukin-6 and interleukin10 levels in chronic lymphocytic leukemia: correlation with phenotypic characteristics and outcome. Blood. 97:256-263.
6. Hahne, M., et al. 1996. Melanoma cell expression of Fas(Apo-1/CD95) ligand: implications for tumor immune escape. Science. 274:1363-1366.

7. Tinhofer, I., et al. 1998. Differential sensitivity of CD4+ and CD8+ T lymphocytes to the killing efficacy of Fas (Apo-1/CD95) ligand+ tumor cells in B chronic lymphocytic leukemia. Blood. 91:4273-4281.

8. Cantwell, M., Hua, T., Pappas, J., and Kipps, T.J 1997. Acquired CD40-ligand deficiency in chronic lymphocytic leukemia. Nat. Med. 3:984-989.

9. de Totero, D., et al. 1999. IL4 production and increased CD30 expression by a unique CD8+ Tcell subset in B-cell chronic lymphocytic leukaemia. Br. J. Haematol. 104:589-599.

10. Kay, N.E., Han, L., Bone, N., and Williams, G. 2001. Interleukin 4 content in chronic lymphocytic leukaemia (CLL) B cells and blood CD8+ T cells from B-CLL patients: impact on clonal B-cell apoptosis. Br. J. Haematol. 112:760-767.

11. Cerutti, A., et al. 2001. Dysregulation of CD30+ T cells by leukemia impairs isotype switching in normal B cells. Nat. Immunol. 2:150-156.

12. Krackhardt, A.M., et al. 2002. T-cell responses against chronic lymphocytic leukemia cells: implications for immunotherapy. Blood. 100:167-173.

13. Rincon, M., Derijard, B., Chow, C.W., Davis, R.J., and Flavell, R.A. 1997. Reprogramming the signalling requirement for AP-1 (activator protein-1) activation during differentiation of precursor CD4+ T-cells into effector Th1 and Th2 cells. Genes Funct. 1:51-68.

14. Dong, C., et al. 1998. Defective T cell differentiation in the absence of Jnk1. Science. 282:2092-2095.

15. Yang, D.D., etal. 1998. Differentiation of CD4+ T cells to Th1 cells requires MAP kinase JNK2. Immunity. 9:575-585.

16. Dan, I., et al. 2000. Molecular cloning of MINK, a novel member of mammalian GCK family kinases, which is up-regulated during postnatal mouse cerebral development. FEBS Lett. 469:19-23.

17. Bachner, D., Sedlacek, Z., Korn, B., Hameister, H., 
and Poustka, A. 1995. Expression patterns of two human genes coding for different rab GDP-dissociation inhibitors (GDIs), extremely conserved proteins involved in cellular transport. Hum. Mol. Genet. 4:701-708.

18. Scheffzek, K., Stephan, I., Jensen, O.N., Illenberger, D., and Gierschik, P. 2000. The Rac-RhoGDI complex and the structural basis for the regulation of Rho proteins by RhoGDI. Nat. Struct. Biol. 7:122-126.

19. Hoffman, G.R., Nassar, N., and Cerione, R.A. 2000. Structure of the Rho family GTP-binding protein $\mathrm{Cdc} 42$ in complex with the multifunctional regulator RhoGDI. Cell. 100:345-356.

20. Schmitz, M.L., Bacher, S., and Dienz, O. 2003 NF-kappaB activation pathways induced by $\mathrm{T}$ cell costimulation. FASEB J. 17:2187-2193.

21. Adams, B.S., et al. 1992. Localization of the gene encoding R kappa B (NFRKB), a tissue-specific DNA binding protein, to chromosome 11q24-q25. Genomics. 14:270-274.

22. Conner, S.D., and Schmid, S.L. 2002. Identification of an adaptor-associated kinase, AAK1, as a regulator of clathrin-mediated endocytosis. J. Cell Biol. 156:921-929.

23. Faundez, V., Horng, J.T., and Kelly, R.B. 1998. A function for the AP3 coat complex in synaptic vesicle formation from endosomes. Cell. 93:423-432.

24. Rossmann, E.D., Lewin, N., Jeddi-Tehrani, M., Osterborg, A., and Mellstedt, H. 2002. Intracellular $\mathrm{T}$ cell cytokines in patients with $\mathrm{B}$ cell chronic lymphocytic leukaemia (B-CLL). Eur. J. Haematol. 68:299-306.

25. Krugmann, S., et al. 2002. Identification of ARAP3, a novel PI3K effector regulating both Arf and Rho GTPases, by selective capture on phosphoinositide affinity matrices. Mol. Cell. 9:95-108.

26. Post, P.L., Bokoch, G.M., and Mooseker, M.S. 1998. Human myosin-IXb is a mechanochemically active motor and a GAP for rho. J. Cell Sci. 111:941-950.

27. Yayoshi-Yamamoto, S., Taniuchi, I., and Watanabe, T. 2000. FRL, a novel formin-related protein, binds to Rac and regulates cell motility and survival of macrophages. Mol. Cell. Biol. 20:6872-6881.

28. Blanchoin, L., Pollard, T.D., and Mullins, R.D. 2000. Interactions of ADF/cofilin, $\mathrm{Arp} 2 / 3 \mathrm{com}$ plex, capping protein and profilin in remodeling of branched actin filament networks. Curr. Biol. 10:1273-1282.

29. Stowers, L., Yelon, D., Berg, L.J., and Chant, J. 1995. Regulation of the polarization of $\mathrm{T}$ cells toward antigen-presenting cells by Ras-related GTPase CDC42. Proc. Natl. Acad. Sci. U. S. A. 92:5027-5031.

30. Labno, C.M., et al. 2003. Itk functions to control actin polymerization at the immune synapse through localized activation of Cdc42 and WASP. Curr. Biol. 13:1619-1624.

31. Buday, L., Wunderlich, L., and Tamas, P. 2002. The Nck family of adapter proteins: regulators of actin cytoskeleton [review]. Cell. Signal. 14:723-731.

32. Yamauchi,J., Nagao, M., Kaziro, Y., and Itoh, H. 1997. Activation of $\mathrm{p} 38$ mitogen-activated protein kinase by signaling through $G$ protein-coupled receptors. Involvement of Gbetagamma and Galphaq/ 11 subunits. J. Biol. Chem. 272:27771-27777.

33. Frost, J.A., Xu, S., Hutchison, M.R., Marcus, S, and Cobb, M.H. 1996. Actions of Rho family small G proteins and p21-activated protein kinases on mitogen-activated protein kinase family members. Mol. Cell. Biol. 16:3707-3713.

34. Muller, M.R., Tsakou, G., Grunebach, F., Schmidt, S.M., and Brossart, P. 2004. Induction of chronic lymphocytic leukemia (CLL)-specific CD4- and CD8-mediated T-cell responses using RNA-transfected dendritic cells. Blood. 103:1763-1769.

35. Gitelson, E., et al. 2003. Chronic lymphocytic leukemia-reactive $\mathrm{T}$ cells during disease progression and after autologous tumor cell vaccines. Clin. Cancer Res. 9:1656-1665.

36. Rincon, M., et al. 1998. Interferon-gamma expression by Th1 effector T cells mediated by the p38 MAP kinase signaling pathway. EMBO J. 17:2817-2829.

37. Yamashita, M., et al. 1999. T cell antigen receptor-mediated activation of the Ras/mitogenactivated protein kinase pathway controls interleukin 4 receptor function and type-2 helper $\mathrm{T}$ cell differentiation. Proc. Natl. Acad. Sci. U. S. A. 96:1024-1029.

38. Dron, M., et al. 2002. Molecular cloning of ADIR, a novel interferon responsive gene encoding a protein related to the torsins. Genomics. 79:315-325

39. Bromley, S.K., et al. 2001. The immunological synapse and CD28-CD80 interactions. Nat. Immunol. 2:1159-1166

40. Stinchcombe, J.C., Bossi, G., Booth, S., and Griffiths, G.M. 2001. The immunological synapse of CTL contains a secretory domain and membrane bridges. Immunity. 15:751-761.

41. Waugh, S.M., Harris, J.L., Fletterick, R., and Craik, C.S. 2000. The structure of the pro-apoptotic protease granzyme B reveals the molecular determinants of its specificity. Nat. Struct. Biol. 7:762-765.
42. Baker, E., Sayers, T.J., Sutherland, G.R., and Smyth, M.J. 1994. The genes encoding NK cell granule serine proteases, human tryptase-2 (TRYP2) and human granzyme A (HFSP), both map to chromosome 5q11-q12 and define a new locus for cytotoxic lymphocyte granule tryptases. Immunogenetics. 40:235-237.

43. Radoja, S., et al. 2001. CD8(+) tumor-infiltrating $\mathrm{T}$ cells are deficient in perforin-mediated cytolytic activity due to defective microtubule-organizing center mobilization and lytic granule exocytosis. J. Immunol. 167:5042-5051.

44. Radoja, S., Saio, M., and Frey, A.B. 2001. CD8+ tumor-infiltrating lymphocytes are primed for Fasmediated activation-induced cell death but are not apoptotic in situ. J. Immunol. 166:6074-6083.

45. Sabapathy, K., et al. 2001. c-Jun NH2-terminal kinase (JNK) 1 and JNK2 have similar and stagedependent roles in regulating $\mathrm{T}$ cell apoptosis and proliferation. J. Exp. Med. 193:317-328.

46. Bojarska-Junak, A., Rolinski, J., Wasik-Szczepaneko, E., Kaluzny, Z., and Dmoszynska, A. 2002. Intracellular tumor necrosis factor production by $\mathrm{T}$ - and B-cells in B-cell chronic lymphocytic leukemia. Haematologica. 87:490-499.

47. Fiorentino, D.F., et al. 1991. IL-10 acts on the antigen-presenting cell to inhibit cytokine production by Th1 cells. J. Immunol. 146:3444-3451.

48. Willems, F., et al. 1994. Interleukin-10 inhibits B7 and intercellular adhesion molecule-1 expression on human monocytes. Eur. J. Immunol. 24:1007-1009.

49. Takayama, T., et al. 2001. Mammalian and viral IL-10 enhance C-C chemokine receptor 5 but downregulate $\mathrm{C}-\mathrm{C}$ chemokine receptor 7 expression by myeloid dendritic cells: impact on chemotactic responses and in vivo homing ability. J. Immunol. 166:7136-7143.

50. Sozzani, S., et al. 1998. Interleukin 10 increases CCR5 expression and HIV infection in human monocytes. J. Exp. Med. 187:439-444.

51. Bichi, R., et al. 2002. Human chronic lymphocytic leukemia modeled in mouse by targeted TCL1 expression. Proc. Natl. Acad. Sci. U. S. A. 99:6955-6960.

52. Mutter, G.L., et al. 2001. Global expression changes of constitutive and hormonally regulated genes during endometrial neoplastic transformation. Gynecol. Oncol. 83:177-185.

53. Li, C., and Wong, W.H. 2001. Model-based analysis of oligonucleotide arrays: expression index computation and outlier detection. Proc. Natl. Acad. Sci.U.S. A. 98:31-36. 